\title{
In Silico Investigation into Cellular Mechanisms of Cardiac Alternans in Myocardial Ischemia
}

\author{
Jiaqi Liu, ${ }^{1}$ Yinglan Gong, ${ }^{1}$ Ling Xia, ${ }^{1}$ and Xiaopeng Zhao ${ }^{2}$ \\ ${ }^{1}$ Department of Biomedical Engineering, Zhejiang University, Hangzhou 310027, China \\ ${ }^{2}$ Department of Mechanical, Aerospace, and Biomedical Engineering, University of Tennessee, Knoxville, TN 37996, USA \\ Correspondence should be addressed to Yinglan Gong; yinglangong@zju.edu.cn and Ling Xia; xialing@zju.edu.cn
}

Received 15 August 2016; Accepted 9 November 2016

Academic Editor: Michele Migliore

Copyright (c) 2016 Jiaqi Liu et al. This is an open access article distributed under the Creative Commons Attribution License, which permits unrestricted use, distribution, and reproduction in any medium, provided the original work is properly cited.

\begin{abstract}
Myocardial ischemia is associated with pathophysiological conditions such as hyperkalemia, acidosis, and hypoxia. These physiological disorders may lead to changes on the functions of ionic channels, which in turn form the basis for cardiac alternans. In this paper, we investigated the roles of hyperkalemia and calcium handling components played in the genesis of alternans in ischemia at the cellular level by using computational simulations. The results show that hyperkalemic reduced cell excitability and delayed recovery from inactivation of depolarization currents. The inactivation time constant $\tau_{f}$ of L-type calcium current $\left(I_{\mathrm{CaL}}\right)$ increased obviously in hyperkalemia. One cycle length was not enough for $I_{\mathrm{CaL}}$ to recover completely. Alternans developed as a result of $I_{\mathrm{CaL}}$ responding to stimulation every other beat. Sarcoplasmic reticulum calcium-ATPase (SERCA2a) function decreased in ischemia. This change resulted in intracellular $\mathrm{Ca}\left(\mathrm{Ca}_{i}\right)$ alternans of small magnitude. A strong $\mathrm{Na}^{+}-\mathrm{Ca}^{2+}$ exchange current $\left(I_{\mathrm{NCX}}\right)$ increased the magnitude of $\mathrm{Ca}_{i}$ alternans, leading to APD alternans through excitation-contraction coupling. Some alternated repolarization currents contributed to this repolarization alternans.
\end{abstract}

\section{Introduction}

The mechanisms underlying ventricular arrhythmias are complex [1]. Ischemia is one of the main causes. Cardiac arrhythmias are produced by electrophysiological disturbances of the heart [1]. Three major pathophysiological conditions linked to acute myocardial ischemia have been identified, including elevated extracellular potassium, acidosis, and anoxia [2]. These conditions cause changes of electrical activities that produce the potent arrhythmia substrate.

T-wave alternans (TWA) can be used for predicting arrhythmogenesis in clinical practice [3]. TWA refers to beatto-beat alternation in the morphology and amplitude of the ST-segment or T-wave magnitude [3]. Electrical instabilities in ischemia promote the occurrence of TWA. Animal experiments show that ischemia increases the magnitude of TWA [3]. Moreover, TWA alone can be identified as a strong indicator for ischemic cardiomyopathy [4]. It originates from action potential duration (APD) alternans at the cellular level [3].

To understand the mechanism of TWA, the study of APD alternans is necessary. APD alternans can be caused either by voltage instabilities (voltage-driven alternans) or by $\mathrm{Ca}^{2+}$ handling dynamics instabilities $\left(\mathrm{Ca}^{2+}\right.$-driven alternans) or their interactions [5]. Because of the bidirectional coupling between membrane voltage kinetics and Ca handling dynamics, it is difficult to identify the exact mechanism of APD alternans $[6,7]$. Voltage instabilities or $\mathrm{Ca}^{2+}$ handling instabilities affect alternans occurring through changes of ionic currents. Thus, there must exist ionic basis in the genesis of alternans. In order to explore the role of ionic currents in the genesis of alternans, computational simulation methods are applied $[8,9]$. Eleven factors have been experimentally reported to be related to cardiac alternans [8]. In order to find out the most relevant factors, investigators compared the differences of these factors between normal and alternans groups [8]. There are significant differences in the following 6 ionic currents between the two groups: the fast sodium current $\left(I_{\mathrm{Na}}\right)$, the Ltype calcium current $\left(I_{\mathrm{CaL}}\right)$, the rapid delayed rectifier potassium current, the sodium calcium exchange current $\left(I_{\mathrm{NCX}}\right)$, the sarcoplasmic reticulum (SR) calcium release current $\left(I_{\text {rel }}\right)$, and the SR calcium reuptake current $\left(I_{\text {up }}\right)[8,9]$. These 6 
currents play an important role in the development of alternans. Voltage-driven alternans is related to APD restitution properties $[1,9]$. APD restitution curve results from collective effects of the recovery properties of all the ionic currents and their interactions with membrane voltage $[1,5]$. Sarcolemmal $\mathrm{K}^{+}$and $\mathrm{Ca}^{2+}$ currents have an influence in the genesis of voltage-driven alternans. Transmembrane proteins such as $\mathrm{Na}^{+}-\mathrm{Ca}^{2+}$ exchange and $\mathrm{Na}^{+}-\mathrm{K}^{+}$pump also take an effect [8]. $\mathrm{Ca}_{i}$ alternans is subsequently induced by the effect of voltagedependent $I_{\mathrm{CaL}}$ current $[6,7] . \mathrm{Ca}^{2+}$-driven alternans originates from steep fractional $\mathrm{Ca}^{2+}$ release relationship [10] or a generic mechanism of RyR properties, refractoriness, randomness, and recruitment [11]. $I_{\text {rel }}, I_{\text {up }}, I_{\mathrm{CaL}}$, and $I_{\mathrm{NCX}}$ have an effect on the genesis of $\mathrm{Ca}^{2+}$-driven alternans [6, 7]. A strong $I_{\mathrm{NCX}}$ can translate $\mathrm{Ca}_{i}$ alternans to voltage alternans [12].

Some of the 6 factors are related to instabilities of electrical activities in ischemia. Intracellular and extracellular acidosis affect ionic currents as channel proteins function like enzymes [13]. Conductibility of $I_{\mathrm{Na}}$ and $I_{\mathrm{CaL}}$ is decreased by acidosis. $I_{\text {rel }}$ current is reduced significantly by acidosis [13]. SERCA2a is regulated by energy metabolism and its function is greatly decreased in ischemia [14]. A population-based study shows that the conductance $g_{\mathrm{CaL}}$ contributes most to the occurrence of APD alternans. Under ischemic conditions, there are also other currents such as $I_{\mathrm{NCX}}, I_{\mathrm{Kr}}$, and $I_{\mathrm{Ks}}$ that play a role [15].

Pathophysiological conditions in ischemia, such as hyperkalemia, acidosis, and hypoxia, promote alternans occurrence by affecting ionic currents at the cellular level. While many experimental and numerical studies reveal voltage- or $\mathrm{Ca}^{2+}$-dependent cellular mechanism, how ischemic conditions cause alternans remains unclear. In this work, we aim to investigate how electrical changes in ischemia promote alternans using computer simulations.

\section{Methods}

2.1. Hyperkalemic Condition. The epicardial ten Tusscher model (TNNP) [16] was employed in this study. In the epicardial cell model, we simulated hyperkalemic condition by increasing the extracellular potassium concentration $\left(\left[\mathrm{K}^{+}\right]_{\mathrm{o}}\right)$. We changed $\left[\mathrm{K}^{+}\right]_{\mathrm{o}}$ alone to investigate its independent role in the development of alternans. $\left[\mathrm{K}^{+}\right]_{\mathrm{o}}$ concentration was set to increase from 5.4 to $15 \mathrm{mM}$. Cycle length was applied at $400 \mathrm{~ms}$.

2.2. SERCA2a Function Decreased in Ischemia. A thermodynamic model of the cardiac SERCA2a [17] was integrated into the TNNP model. The thermodynamic model based on biophysical kinetic is sensitive to metabolism compromised in ischemia.

$$
\begin{aligned}
2 \mathrm{Ca}_{i}^{2+} & +\mathrm{MgATP}+\mathrm{H}_{2} \mathrm{O} \\
& \Longleftrightarrow 2 \mathrm{Ca}_{\mathrm{sr}}^{2+}+\mathrm{MgADP}+\mathrm{Pi}+\mathrm{H}^{+}
\end{aligned}
$$

The process of Ca uptake from the cytoplasm to SR can be presented by the above reaction equation. The equation shows that translating two $\mathrm{Ca}^{2+}$ needs the hydrolysis of one

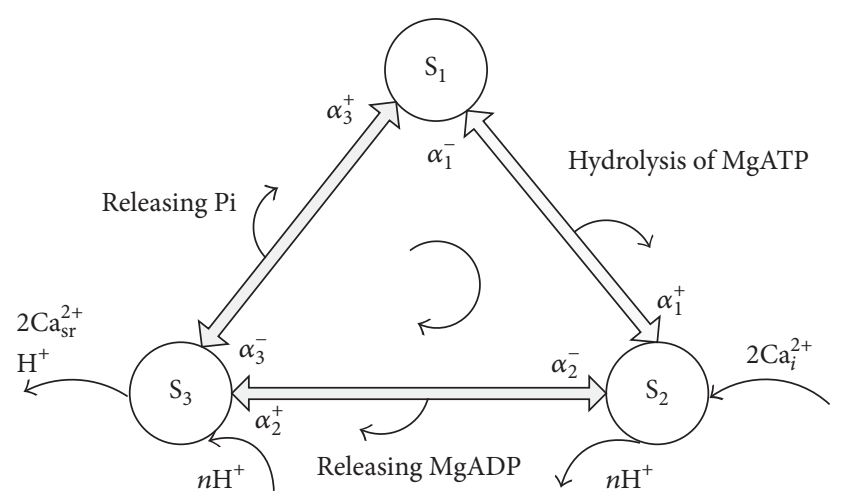

FIGURE 1: Schematic of the three-state model. $S_{1}, S_{2}$, and $S_{3}$ represent the state of SERCA pump in the reaction process. The rate constants are represented by $\alpha_{i}^{ \pm}(1,2,3)$ and signs represent the forward or backward direction of reaction. Hydrolysis of MgATP and the release of $\mathrm{MgADP} / \mathrm{Pi}$ occur in the positive direction during the transformation process. The reaction process is simplified from the E1-E2 model [18, 19].

ATP. At the same time, the products $\mathrm{MgADP}, \mathrm{Pi}$, and $\mathrm{H}^{+}$are released. This reversible reaction is modeled by E1-E2 model $[18,19]$ which consisted of two conformational changes of $\mathrm{Ca}^{2+}$-binding sites.

The cardiac SERCA2a model applied in our study was a three-state model. The three-state model (Figure 1) is simplified from the E1-E2 model [18, 19] using rapid equilibrium assumption. In the positive direction, state $S_{1}$ transforms to $S_{2}$ via the hydrolysis of ATP. State $\mathrm{S}_{2}$ indicates $\mathrm{Ca}^{2+}$-binding sites binding $\mathrm{Ca}^{2+}$ and the $\mathrm{Ca}^{2+} / \mathrm{H}^{+}$countertransport transporting $\mathrm{H}^{+}$from SR to the cytoplasm, state $\mathrm{S}_{3}$ represents the $\mathrm{Ca}^{2+}$ binding sites releasing $\mathrm{Ca}^{2+}$ to the $\mathrm{SR}$ and $\mathrm{Ca}^{2+} / \mathrm{H}^{+}$countertransport binding $\mathrm{H}^{+}$to SERCA. The rate constants $\left(\alpha_{i}\right)$ are functions of intracellular $\mathrm{Pi}, \mathrm{ATP}, \mathrm{ADP}$, and $\mathrm{H}^{+}$concentrations. See Appendix for formulas of these rate constants [17].

$$
V_{\text {cycle }}=\frac{\alpha_{1}^{+} \alpha_{2}^{+} \alpha_{3}^{+}-\alpha_{1}^{-} \alpha_{2}^{-} \alpha_{3}^{-}}{\Sigma},
$$

where $V_{\text {cycle }}$ is clockwise cycle rate per pump at steady state. By modifying physiological parameters we could simulate metabolism compromised. In ischemia $\mathrm{pH}$ was decreased accompanied with a decrease of intracellular ATP. We set $\mathrm{pH}$ at 6 and ATP concentration at $4.2 \mathrm{mM}$. At the same time the concentrations of intracellular ADP and Pi [20] were simulated to increase to $100 \mathrm{nM}$ and $30 \mathrm{mM}$ [21], respectively. Values of other parameters in the formulas were the same as in the original three-state model [17].

$$
I_{\text {up }}=N * V_{\text {cycle }} \text {. }
$$

The value of $I_{\text {up }}$ was in proportion to the whole-cell pump flux. The whole-cell pump flux was determined by $V_{\text {cycle }}$ and the numbers of SERCA pumps on the SR membrane. In order to calculate $I_{\text {up }}$ under compromised metabolism conditions, we multiplied $V_{\text {cycle }}$ by the constant $N$ as a scale factor. The value of $N$ was the ratio of maximum $I_{\text {up }}$ obtained by original TNNP model simulation and maximum $V_{\text {cycle }}$ under normal conditions. 


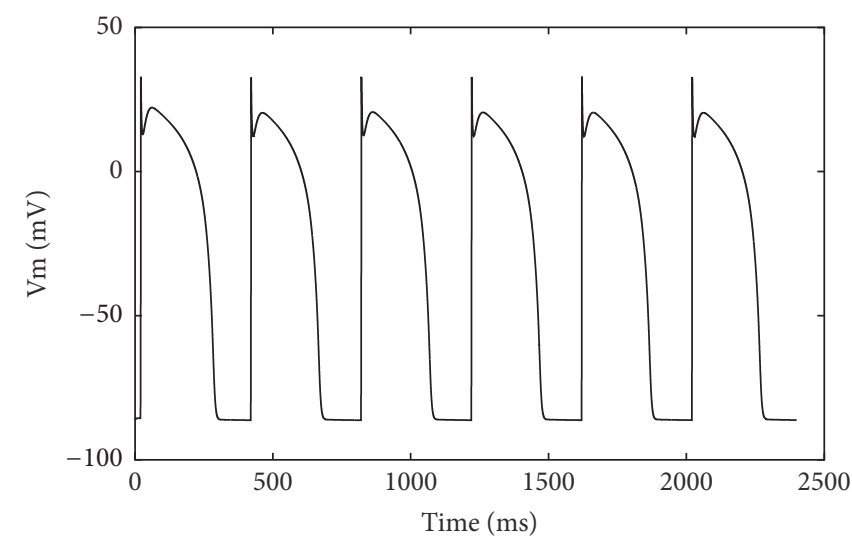

(a)

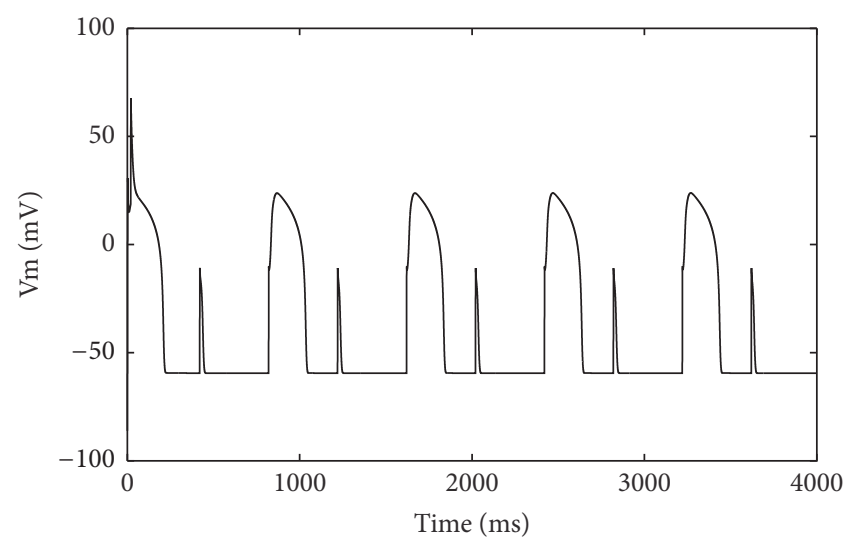

(b)

FIgURE 2: APD computed at the cycle length of $400 \mathrm{~ms}$. (a) APs in control condition with $\left[\mathrm{K}^{+}\right]_{\mathrm{o}}=5.4 \mathrm{mM}$. (b) Alternate APs in hyperkalemia with $\left[\mathrm{K}^{+}\right]_{\mathrm{o}}=15 \mathrm{mM}$.

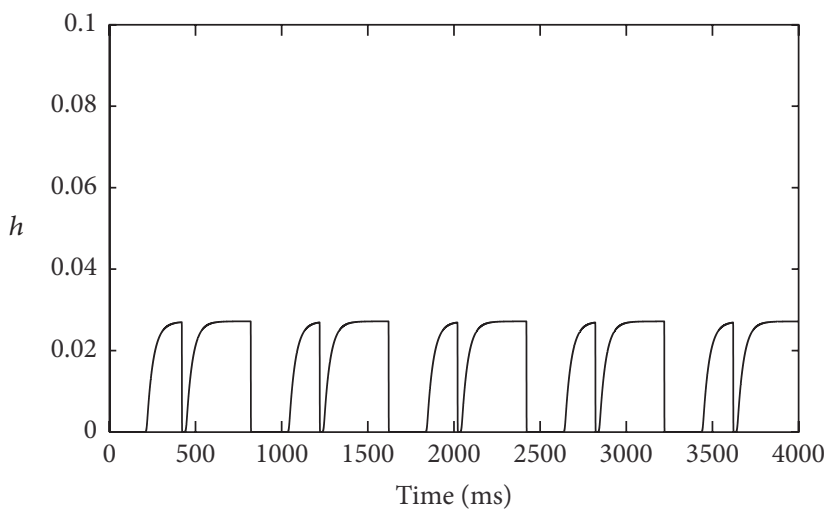

(a)

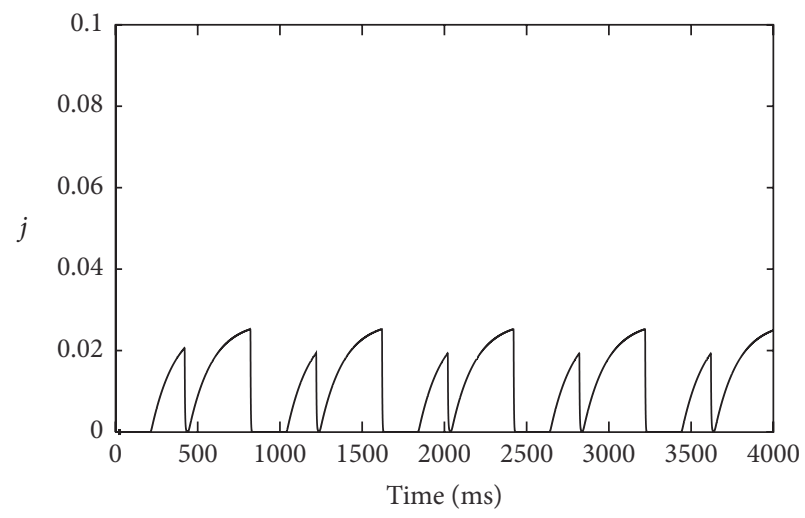

(b)

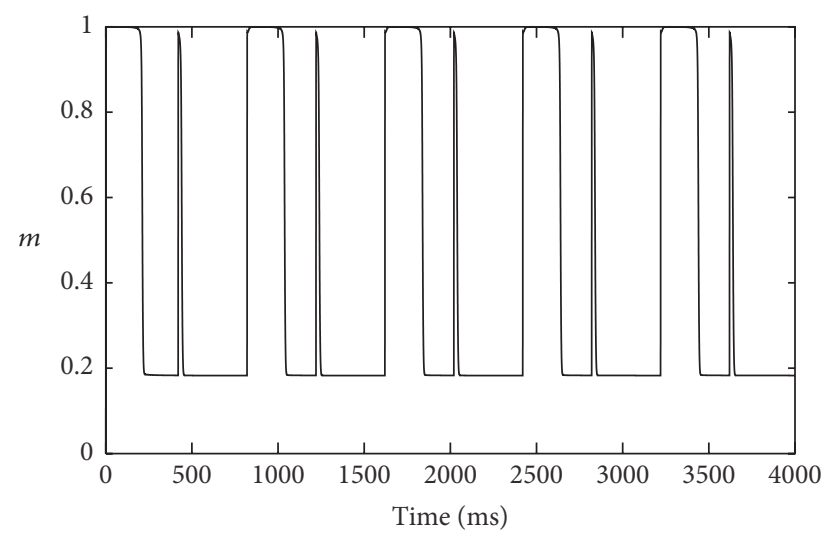

(c)

FIgURE 3: Gating variables of $I_{\mathrm{Na}}$ in hyperkalemia. (a) Fast inactivation gate $h$; (b) slow inactivation gate $j$; (c) activation gate $m$.

\section{Results}

3.1. The Effects of Hyperkalemia on APD and Ionic Currents. While the cycle length was applied at $400 \mathrm{~ms}$, no APD alternans existed under normal conditions (Figure 2(a)). There existed no alternans except for the elevated resting potential and decreased amplitude of action potential when the $\left[\mathrm{K}^{+}\right]_{\mathrm{o}}$ values were in between $5 \mathrm{mM}$ and $14.7 \mathrm{mM}$. APD alternans occurred in hyperkalemia with $\left[\mathrm{K}^{+}\right]_{0}$ ranging from 14.7 to $15 \mathrm{mM}$. The $\left[\mathrm{K}^{+}\right]_{\mathrm{o}}$ values in this range correspond to severe hyperkalemia. Moreover, significantly elevated $\left[\mathrm{K}^{+}\right]_{\mathrm{o}}$ values may also occur in ischemic hearts as well as in isolated hearts in experiments. The longer AP manifested two depolarization phases. These two depolarization phases were 


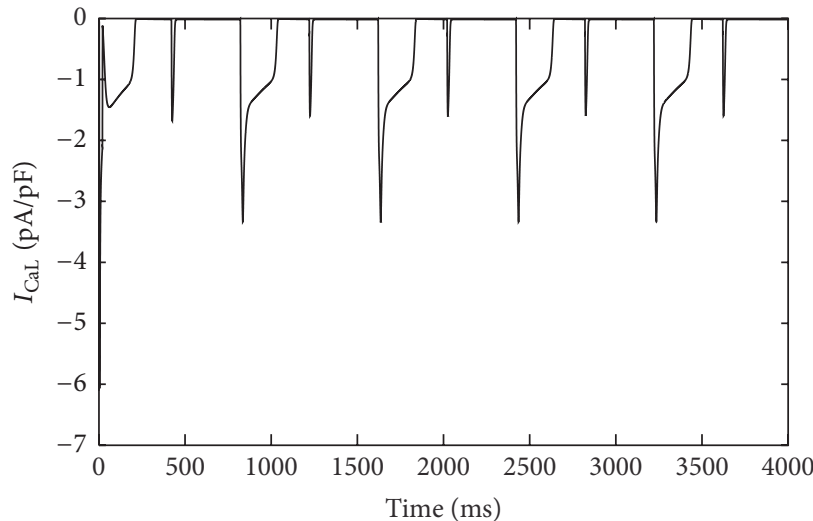

(a)

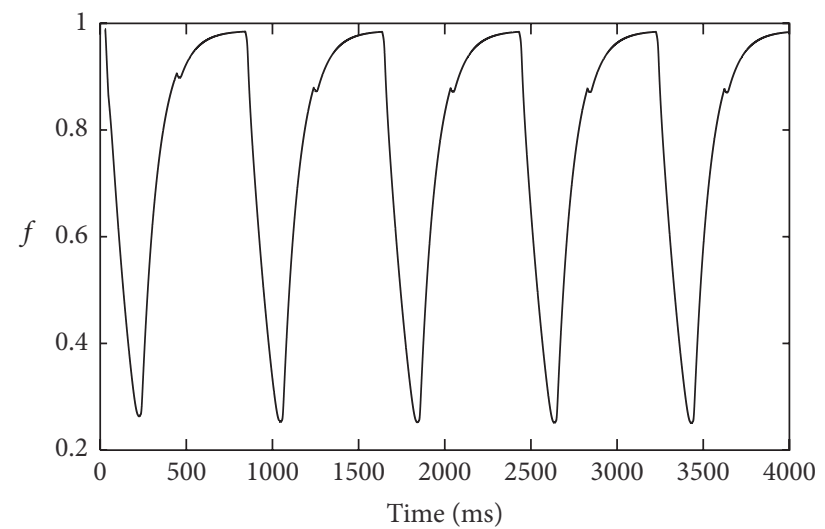

(c)

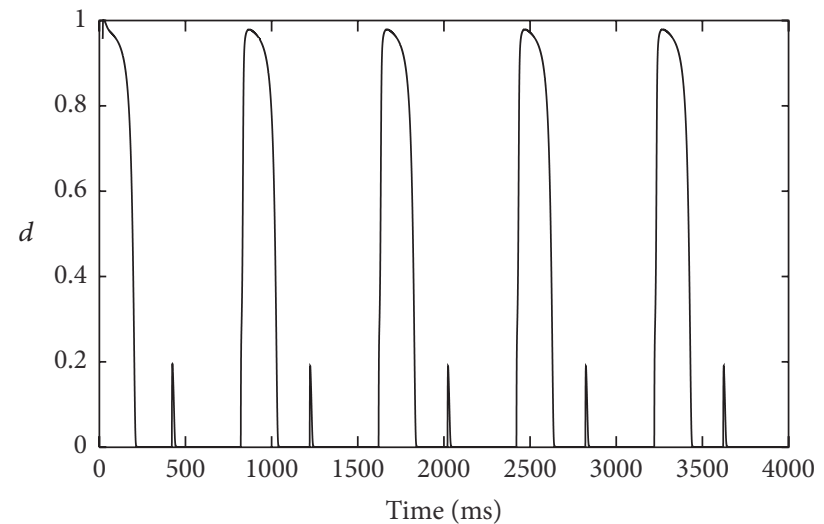

(b)

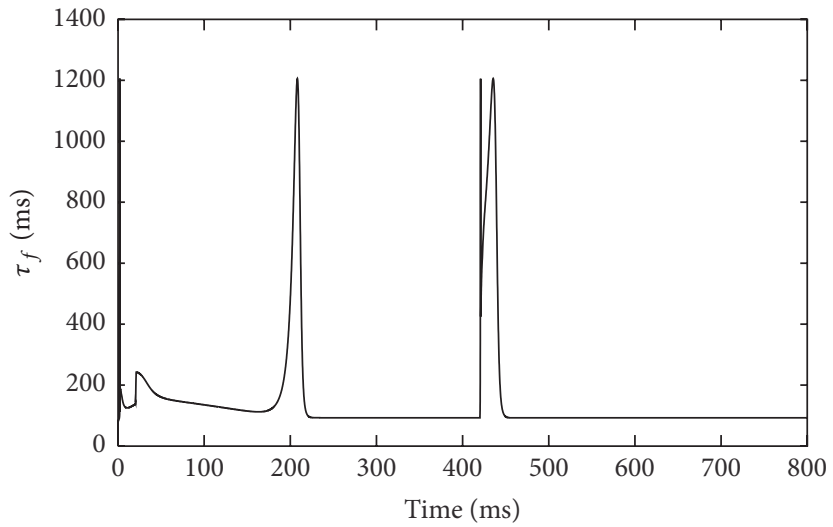

(d)

FIGURE 4: $I_{\mathrm{CaL}}$ and its gating variables in hyperkalemia. (a) $I_{\mathrm{CaL}}$ current; (b) voltage-dependent activation gate $d$; (c) voltage-dependent inactivation gate $f$; (d) inactivation time constant $\tau_{f}$ of the gate $f$ during two consecutive beats.

maintained by $I_{\mathrm{Na}}$ and $I_{\mathrm{CaL}}$. The availability of $I_{\mathrm{CaL}}$ in shorter APs was reduced, resulting in small depolarization phases during the next beats (Figure 2(b)).

To investigate the process of alternans occurring, depolarization currents were selected to be studied. $I_{\mathrm{Na}}$ decreased significantly in hyperkalemia. Open possibilities of inactivation gates, $h$ and $j$, came near to be zero (Figures 3(a) and $3(b))$. In contrast, the open possibility of activation gate $m$ increased at depolarized resting voltage (Figure 3(c)).

The amplitude of $I_{\mathrm{CaL}}$ showed alternans (Figure 4(a)). Activation gate $d$ was voltage-dependent and manifested alternans from beat to beat (Figure 4(b)). The intracellular calcium-dependent inactivation gate, $f_{\mathrm{ca}}$, was nearly in closed state. Voltage-dependent inactivation gate $f$ needed two cycle lengths to recover completely (Figure 4(c)). Moreover, the inactivation time constant $\tau_{f}$ of the gate $f$ became larger during shorter APs (Figure 4(d)). That further verified the gate $f$ could not recover instantly from inactivation, leading to decreased availability of $I_{\mathrm{CaL}}$ during shorter APs. While $\tau_{f}$ was decreased by $70 \mathrm{~ms}$ (Figure 5(a)), the gate $f$ recovered instantly (Figure 5(b)) and alternans in APD disappeared (Figure 5(c)).

3.2. The Effects of $I_{\mathrm{up}}$ and $I_{\mathrm{NCX}}$ on Ca Transient and APD. As the component of cardiac Ca handling, $I_{\text {up }}$ decreased under ischemic conditions. This change was simulated by modifying the physiological parameters of the SERCA pump model [17]. Thus the direct role of $I_{\text {up }}$ in the onset of $\mathrm{Ca}_{i}$ alternans could be investigated. Decreased $I_{\text {up }}$ slowed down the rate of SR Ca uptake and could not balance $\mathrm{Ca}^{2+}$ flux released from SR. As Figure 6(b) showed, $\mathrm{Ca}^{2+}$ transients alternated obviously during early beats and reached a steady state finally. In contrast to alternate $\mathrm{Ca}^{2+}$ transients, APD remained unchanged (Figure 6(a)).

$I_{\mathrm{NCX}}$ decreased under acidic conditions [13]. Decreased $I_{\mathrm{NCX}}$ was also added in the simulation after investigating the effect of $I_{\text {up }}$ on $\mathrm{Ca}_{i}$ alternans. As Figure 7 showed, the magnitude of $\mathrm{Ca}^{2+}$ transient alternans decreased. The result suggested that decreased $I_{\mathrm{NCX}}$ could inhibit $\mathrm{Ca}_{i}$ alternans. Based on this observation, we expected that $\mathrm{Ca}_{i}$ alternans magnitude would increase as $I_{\mathrm{NCX}}$ current increased. Figure 8(b) confirmed the guess. Results showed that APD alternans was accompanied with $\mathrm{Ca}_{i}$ alternans of large magnitude (Figure 8(a)).

To compare the differences in the durations of repolarization between APs, we placed the 6 beats in the coordinate axes in Figure 9(a). Previous study suggested that $I_{\mathrm{Kr}}$ and $I_{\mathrm{Ks}}$ played a role in the occurrence of APD alternans. We selected $I_{\mathrm{Ks}}$ and $I_{\mathrm{Kr}}$ to investigate their roles in the process. $I_{\mathrm{Kr}}$ and $I_{\mathrm{Ks}}$ alternated from beat to beat as shown in Figures 9(b) and 9(c). 


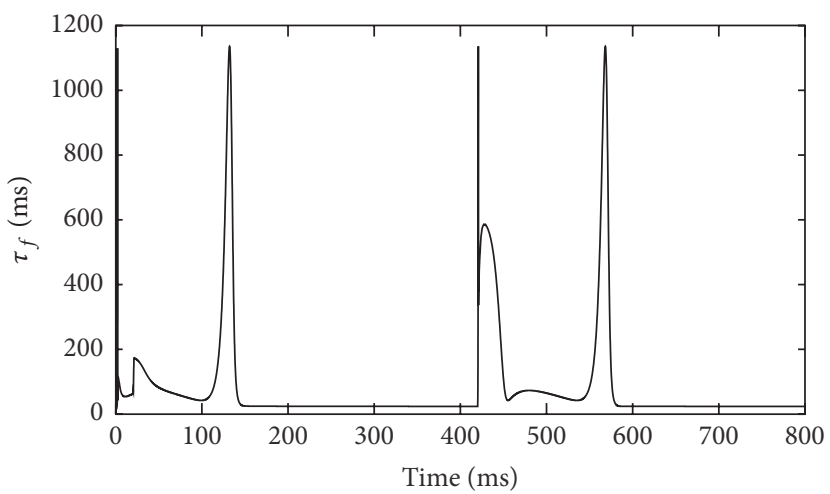

(a)

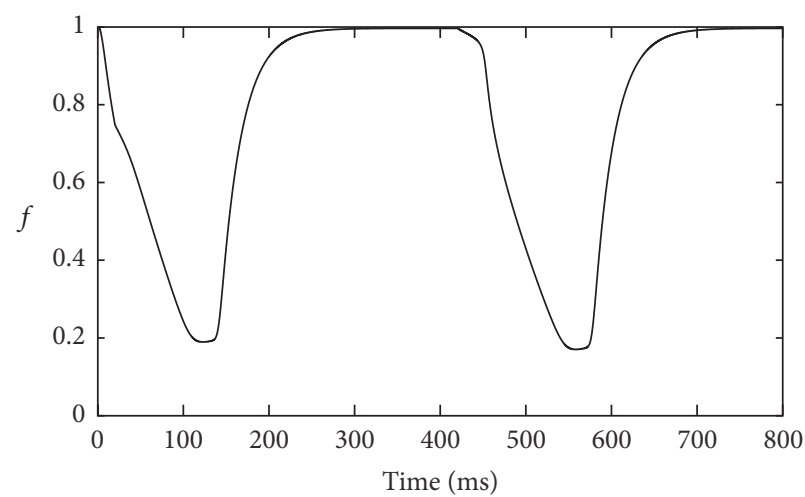

(b)

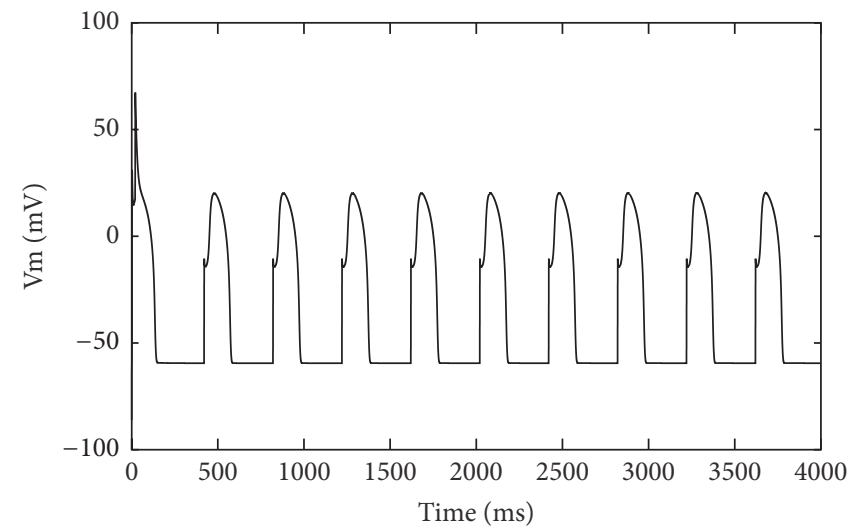

(c)

FIGURE 5: Simulations of the inactivation gate $f$ and APD after decreasing inactivation time constant $\tau_{f}$ in hyperkalemia. (a) Decreased inactivation time constant $\tau_{f}$ of the gate $f$; (b) voltage-dependent inactivation gate $f$. Decreased $\tau_{f}$ made the gate $f$ recover completely before the next APD. The gate $f$ responded once during two consecutive beats in hyperkalemia without decreasing $\tau_{f}$; (c) APs with no alternans.

\section{Discussion}

4.1. The Mechanism of Alternans in Hyperkalemia. Depolarization alternans in hyperkalemia arises from changes in depolarization currents. In order to find out the key factors relating to alternans occurring in hyperkalemia, we selected depolarization currents for analysis. Our simulation results suggest that $I_{\mathrm{Na}}$ is too small to affect the process of depolarization during both longer and shorter APs. $I_{\mathrm{CaL}}$ may be the key factor in the development of alternans. Cycle lengths are fixed and the longer AP is followed by the shorter duration. $I_{\mathrm{CaL}}$ cannot recover completely from inactivation in the shorter duration. Its availability decreases in the following depolarization phase. Thus the next depolarization phase maintained by $I_{\mathrm{Na}}$ alone is small. Small depolarization phase leads to shorter AP. Subsequently, the longer duration provides enough time for $I_{\mathrm{CaL}}$ to recover completely. Shorter APs are following longer APs and alternans develops. In order to further verify the role of $I_{\mathrm{CaL}}, \tau_{f}$ of voltage-dependent inactivation gate $f$ is decreased in simulation. Decreased $\tau_{f}$ indicates that the gate $f$ needs shorter time to recover completely. Then the availability of $I_{\mathrm{CaL}}$ increases in APs. Alternans disappears due to complete response of $I_{\mathrm{CaL}}$ in every beat.
In contrast, some studies investigate alternans mechanisms in ischemia at the tissue level. Previous study supports that the depolarization alternans is linked to conduction abnormalities in the ischemia region [22]. The conduction block occurs under hyperkalemic conditions. Moreover, the depolarization phase is fragmented in the current simulation of hyperkalemia as is consistent with previous observations. Results show that depolarization alternans in ischemia region can be produced by hyperkalemic conditions [23].

Alternate conduction block induced by hyperkalemia leads to APD alternans [24]. The areas of conduction blocks become larger and alternans occurs at slower pacing frequency while increasing the inactivation time constant $\tau_{f}$ [24]. According to the observation, smaller areas are expected to be blocked if $\tau_{f}$ decreases and APD alternans will be depressed in the areas with no block any more. In other words, decreased $\tau_{f}$ can abolish alternans through eliminating conduction block. That is consistent with our observations. Hyperkalemia increases $\tau_{f}$ by depolarizing the resting voltage and thus promotes APD alternans.

4.2. The Direct Role of $I_{\mathrm{up}}$ in $\mathrm{Ca}_{i}$ Alternans. The slow rate of SR Ca uptake contributes to the occurrence of $\mathrm{Ca}_{i}$ alternans 


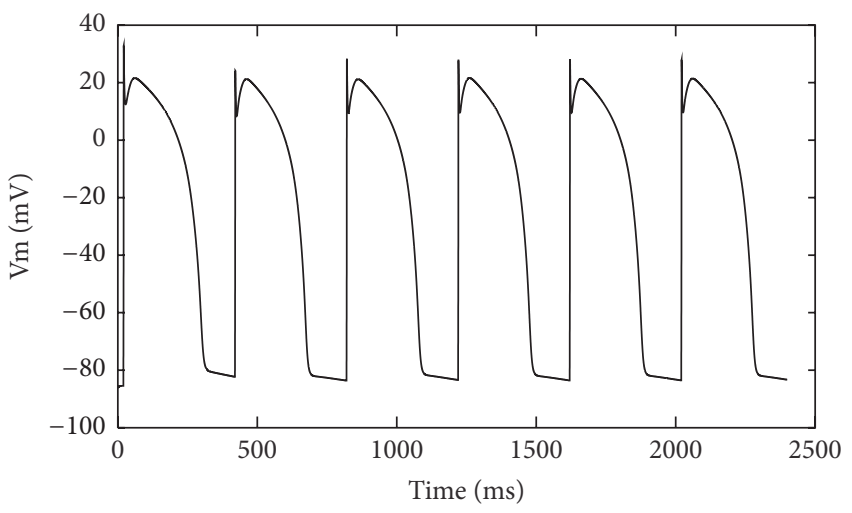

(a)

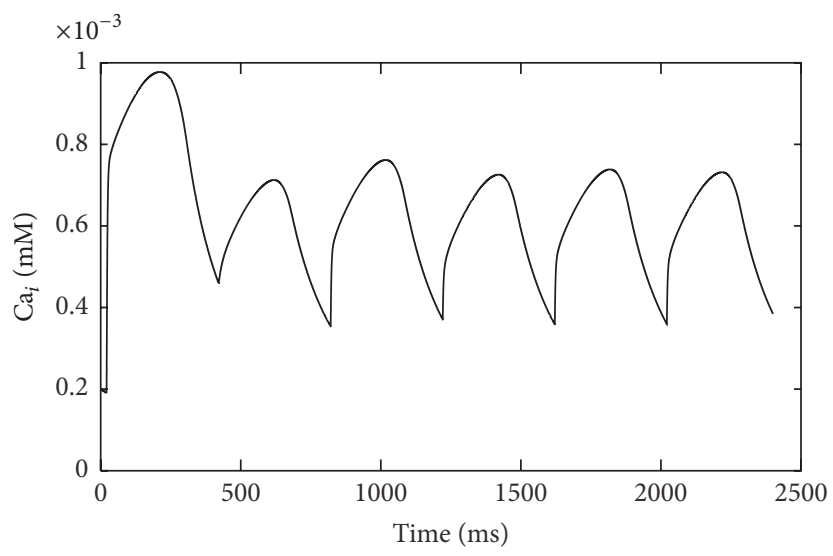

(b)

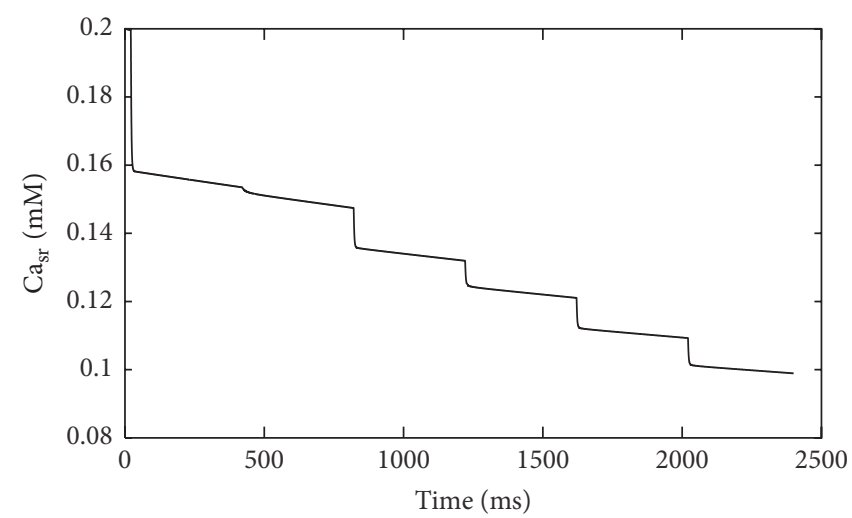

(c)

Figure 6: Ca transient, APs, and $\mathrm{Ca}^{2+}$ content in SR after decreasing $I_{\text {up }}$ current. (a) APs with no alternans; (b) alternate Ca transient; (c) decreased $\mathrm{Ca}^{2+}$ content in SR from beat to beat.

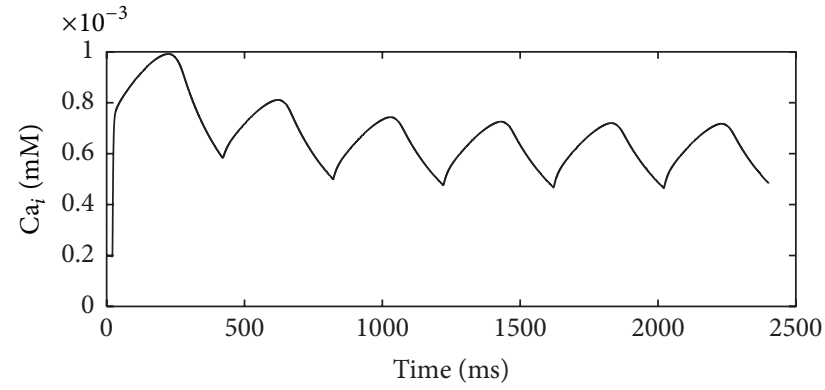

Figure 7: Ca transient after decreasing $I_{\text {up }}$ and $I_{\mathrm{NCX}}$ currents. $\mathrm{Ca}_{i}$ alternans disappeared after decreasing $I_{\mathrm{NCX}}$.

[25]. Qu et al. point out calcium alternans is determined by the interaction of the slopes of the fractional $\mathrm{Ca}^{2+}$ release curve, the SR Ca ${ }^{2+}$ uptake function, and properties of $\mathrm{Ca}^{2+}$ sparks [26]. The independent role of decreased $I_{\text {up }}$ in the $\mathrm{Ca}_{i}$ alternans is investigated in our study. Decreased $I_{\text {up }}$ has no ability to balance $I_{\text {up }}$ current. The Ca content in diastole is affected. Subsequently, the release of $\mathrm{Ca}^{2+}$ from SR is depressed due to elevated $\mathrm{Ca}^{2+}$ in the cytoplasm. Then the
$\mathrm{Ca}$ content in diastole decreases comparing to the last $\mathrm{Ca}$ transient. Fluctuations in cytoplasmic Ca content in diastole originate from unbalance in $\mathrm{Ca}^{2+}$ flux between $I_{\text {up }}$ uptake and $I_{\text {rel }}$ releasing. Transient $\mathrm{Ca}_{i}$ alternans are consequently caused by the fluctuations. According to the unified theory presented by $\mathrm{Qu}$ et al. [26], we could add ischemic changes of $I_{\text {rel }}$ to the cell model to obtain stable calcium alternans. Ca content fluctuation in SR plays a role in producing $\mathrm{Ca}^{2+}$ transients alternans [10]. But our results show that SR load decreases from beat to beat (Figure 6(c)). That suggests SR load may not be the direct factor in the development of $\mathrm{Ca}_{i}$ alternans.

4.3. The Role of $I_{\mathrm{NCX}}$ in the Alternans Translation from $\mathrm{Ca}$ to $A P D$. Larger $I_{\mathrm{NCX}}$ increases $\mathrm{Ca}_{i}$ alternans magnitude. Our results suggest that $\mathrm{Ca}_{i}$ alternans can lead to APD alternans while the $\mathrm{Ca}_{i}$ alternans magnitude is large enough. However, decreased $I_{\text {up }}$ and increased $I_{\mathrm{NCX}}$ are not sufficient to produce stable alternans in our simulations. Previous study also shows that $I_{\mathrm{NCX}}$ is the key factor that translates alternans from $\mathrm{Ca}$ to APD [12]. More precisely, the balance of $I_{\mathrm{NCX}}$ and $I_{\mathrm{Ca}}$ determines coupling in phase of $\mathrm{Ca}_{i}$ alternans to APD alternans [27]. Alternans presented by Wan et al. can arise from the shifted balance of $I_{\mathrm{NCX}}$ and $I_{\mathrm{Ca}}$ at higher pacing 


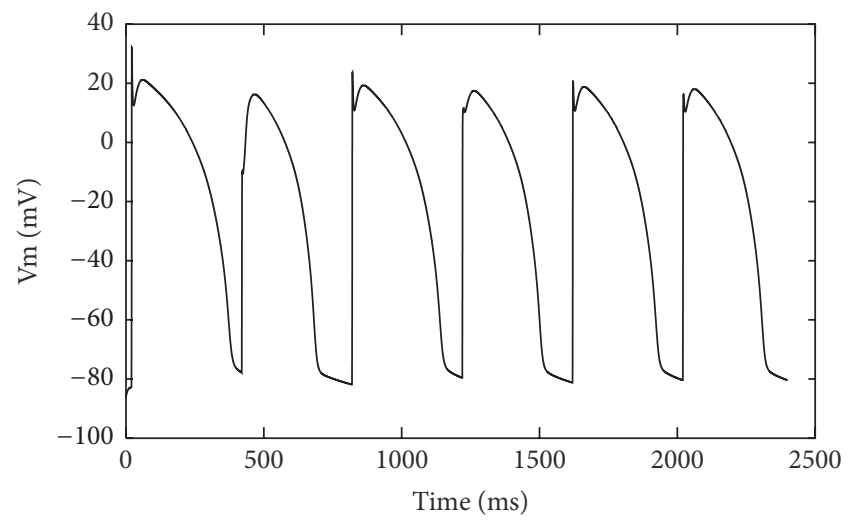

(a)

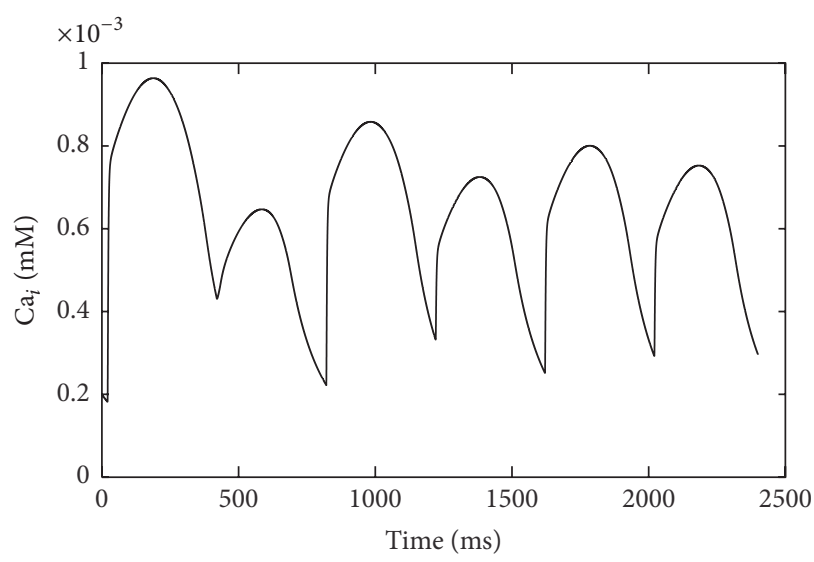

(b)

FIGURE 8: Ca transient and APs after decreasing $I_{\text {up }}$ and increasing $I_{\mathrm{NCX}}$. (a) Alternate APs; (b) $\mathrm{Ca}_{i}$ alternans. Decreased $I_{\text {up }}$ produced Ca alternans; however, the magnitude of $\mathrm{Ca}_{i}$ alternans was larger after increasing $I_{\mathrm{NCX}}$.

rates. In our simulation, the extent of unbalance between these currents shifted by increasing $I_{\mathrm{NCX}}$ at cycle length of $400 \mathrm{~ms}$ could be too small to produce stable alternans [27]. $I_{\mathrm{Kr}}$ and $I_{\mathrm{Ks}}$ contribute to the occurrence of APD alternans in ischemia [15]. $I_{\mathrm{Kr}}$ contributes most due to its larger amplitude.

\section{Conclusion}

In silico simulations have been carried out to investigate cellular mechanisms of cardiac alternans under pathological disorders including hyperkalemia, acidosis, and hypoxia. Pathophysiological changes in ischemia play a significant role in the development of cardiac alternans by affecting ionic currents. Hyperkalemic conditions delay the recovery of depolarization current $I_{\mathrm{CaL}}$. Thus depolarization alternans occurs. Decreased $I_{\text {up }}$ of Ca handling in ischemia promotes $\mathrm{Ca}_{i}$ alternans. A large $I_{\mathrm{NCX}}$ has the ability to translate alternans from Ca to APD. Studying changes of these ionic currents can help further understand cellular mechanisms of the genesis of alternans and form the basis of study of TWA in ischemia.

\section{Appendix}

The rate constants used in (2) are as follows:

$$
\begin{aligned}
& \alpha_{1}^{+}=k_{1}^{+}[\mathrm{MgATP}] \\
& \alpha_{2}^{+}=\frac{k_{2}^{+} \widetilde{\mathrm{Ca}}_{i}^{2}}{\widetilde{\mathrm{C}} \mathrm{a}_{i}^{2+}\left(1+\widetilde{\mathrm{H}}_{i}\right)+\widetilde{\mathrm{H}}_{i}\left(1+\widetilde{\mathrm{H}}_{1}\right)}, \\
& \alpha_{3}^{+}=\frac{k_{3}^{+} \widetilde{\mathrm{H}}_{\mathrm{sr}}}{\widetilde{\mathrm{H}}\left(1+\widetilde{\mathrm{C}} \mathrm{a}_{\mathrm{sr}}^{2}\right)+\widetilde{\mathrm{H}}_{\mathrm{sr}}(1+\widetilde{\mathrm{H}})}, \\
& \alpha_{1}^{-}=\frac{k_{1}^{-} \widetilde{\mathrm{H}}_{i}}{\widetilde{\mathrm{C}} \mathrm{a}_{i}^{2+}\left(1+\widetilde{\mathrm{H}}_{i}\right)+\widetilde{\mathrm{H}}_{i}\left(1+\widetilde{\mathrm{H}}_{1}\right)},
\end{aligned}
$$

$$
\begin{aligned}
& \alpha_{2}^{-}=\frac{k_{2}^{-}[\mathrm{MgADP}] \widetilde{\mathrm{Ca}}_{\mathrm{sr}}^{2} \widetilde{\mathrm{H}}_{\mathrm{sr}}}{\widetilde{\mathrm{H}}\left(1+\widetilde{\mathrm{Ca}}_{\mathrm{sr}}^{2}\right)+\widetilde{\mathrm{H}}_{\mathrm{sr}}(1+\widetilde{\mathrm{H}})}, \\
& \alpha_{3}^{-}=k_{3}^{-}[\mathrm{Pi}],
\end{aligned}
$$

where

$$
\begin{aligned}
\widetilde{\mathrm{Ca}}_{i} & =\frac{\left[\mathrm{Ca}^{2+}\right]_{i}}{K_{d, \mathrm{Ca}_{i}}}, \\
\widetilde{\mathrm{H}}_{i} & =\frac{\left[\mathrm{H}^{+}\right]}{K_{d, \mathrm{H}_{i}}}, \\
\widetilde{\mathrm{H}}_{1} & =\frac{\left[\mathrm{H}^{+}\right]}{K_{d, \mathrm{H}_{1}}}, \\
\widetilde{\mathrm{Ca}}_{\mathrm{sr}} & =\frac{\left[\mathrm{Ca}^{2+}\right]_{\mathrm{sr}}}{K_{d, \mathrm{Ca}_{\mathrm{sr}}}} \\
\widetilde{\mathrm{H}}_{\mathrm{sr}} & =\frac{\left[\mathrm{H}^{+}\right]}{K_{d, \mathrm{H}_{\mathrm{sr}}}}, \\
\widetilde{\mathrm{H}} & =\frac{\left[\mathrm{H}^{+}\right]}{K_{d, \mathrm{H}}} .
\end{aligned}
$$

\section{Competing Interests}

The authors declare that there is no conflict of interests regarding the publication of this paper.

\section{Acknowledgments}

This project is supported in part by the National Natural Science Foundation of China (61527811), a key research grant for national fitness from General Administration of Sports 


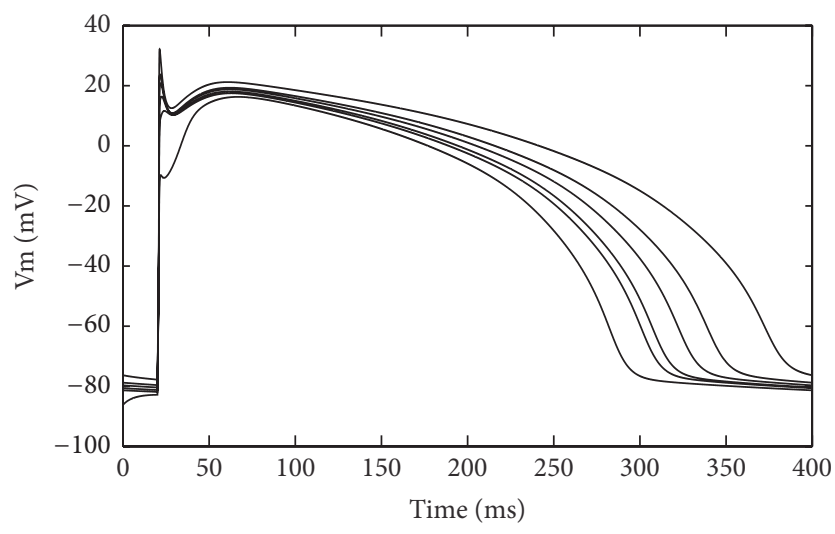

(a)

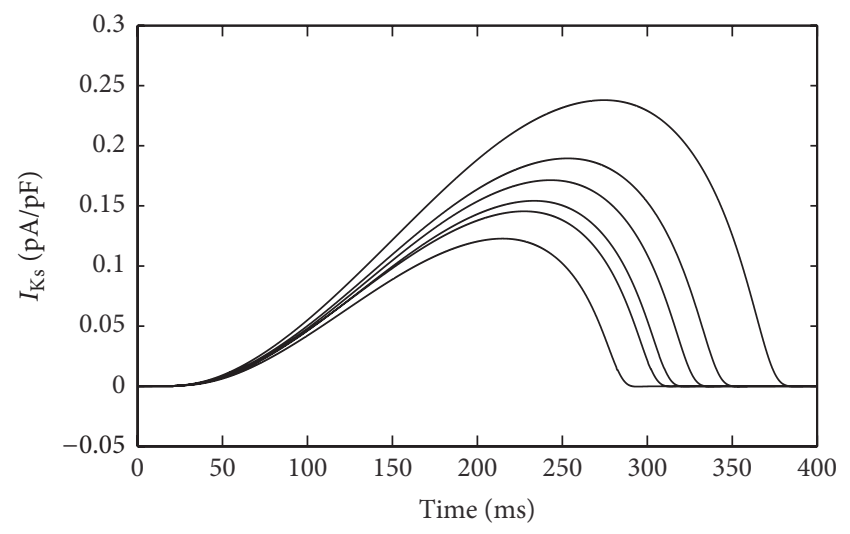

(b)

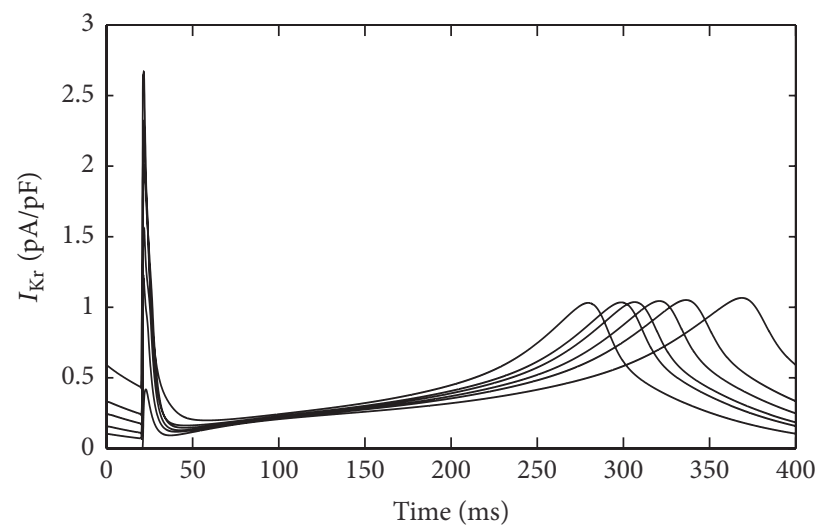

(c)

FIGURE 9: Alternate APs and two repolarization currents in consecutive beats after decreasing Iup and increasing $I_{\mathrm{NCX}}$. (a) Consecutive APs in the same coordinate system. Repolarization alternans was obvious by comparing duration of action potentials; (b) beat-to-beat alternation in repolarization current $I_{\mathrm{Ks}} ;$ (c) beat-to-beat alternation in repolarization current $I_{\mathrm{Kr}}$.

of China (2015B043), and the National Science Foundation (CMMI-1234155).

\section{References}

[1] Z. Qu and J. N. Weiss, "Mechanisms of ventricular arrhythmias: from molecular fluctuations to electrical turbulence," Annual Review of Physiology, vol. 77, pp. 29-55, 2015.

[2] R. M. Shaw and Y. Rudy, "Electrophysiologic effects of acute myocardial ischemia: a theoretical study of altered cell excitability and action potential duration," Cardiovascular Research, vol. 35, no. 2, pp. 256-272, 1997.

[3] R. L. Verrier, T. Klingenheben, M. Malik et al., "Microvolt Twave alternans: physiological basis, methods of measurement, and clinical utility-consensus guideline by international society for Holter and noninvasive Electrocardiology," Journal of the American College of Cardiology, vol. 58, no. 13, pp. 1309-1324, 2011.

[4] T. Chow, D. J. Kereiakes, C. Bartone et al., "Prognostic utility of microvolt T-wave alternans in risk stratification of patients with ischemic cardiomyopathy," Journal of the American College of Cardiology, vol. 47, no. 9, pp. 1820-1827, 2006.

[5] Z. Qu, Y. Xie, A. Garfinkel, and J. N. Weiss, "T-wave alternans and arrhythmogenesis in cardiac diseases," Frontiers in Physiology, vol. 1, article 154, 2010.
[6] D. Sato and C. E. Clancy, "Cardiac electrophysiological dynamics from the cellular level to the organ level," Biomedical Engineering and Computational Biology, vol. 5, pp. 69-75, 2013.

[7] Y.-L. Zang and L. Xia, "Cellular mechanism of cardiac alternans: an unresolved chicken or egg problem," Journal of Zhejiang University: Science B, vol. 15, no. 3, pp. 201-211, 2014.

[8] X. Zhou, A. Bueno-Orovio, M. Orini et al., "Population of human ventricular cell models calibrated with in vivo measurements unravels ionic mechanisms of cardiac alternans," in Proceedings of the IEEE Computing in Cardiology Conference (CinC '13), Zaragoza, Spain, September 2013.

[9] M. L. Koller, S. K. G. Maier, A. R. Gelzer, W. R. Bauer, M. Meesmann, and R. F. Gilmour Jr., "Altered dynamics of action potential restitution and alternans in humans with structural heart disease," Circulation, vol. 112, no. 11, pp. 1542-1548, 2005.

[10] M. E. Díaz, S. C. O’Neill, and D. A. Eisner, "Sarcoplasmic reticulum calcium content fluctuation is the key to cardiac alternans," Circulation Research, vol. 94, no. 5, pp. 650-656, 2004.

[11] R. Rovetti, X. Cui, A. Garfinkel, J. N. Weiss, and Z. Qu, "Spark-induced sparks as a mechanism of intracellular calcium alternans in cardiac myocytes," Circulation Research, vol. 106, no. 10, pp. 1582-1591, 2010.

[12] X. Zhou, A. Bueno-Orovio, M. Orini et al., "In vivo and in silico investigation into mechanisms of frequency dependence of 
repolarization alternans in human ventricular cardiomyocytes," Circulation Research, vol. 118, no. 2, pp. 266-278, 2016.

[13] E. Carmeliet, "Cardiac ionic currents and acute ischemia: from channels to arrhythmias," Physiological Reviews, vol. 79, no. 3, pp. 917-1017, 1999.

[14] S. Krause and M. L. Hess, "Characterization of cardiac sarcoplasmic reticulum dysfunction during short-term, normothermic, global ischemia," Circulation Research, vol. 55, no. 2, pp. 176-184, 1984.

[15] A. M. S. Dutta, J. Walmsley, and B. Rodriguez, "Ionic mechanisms of variability in electrophysiological properties in Ischemia: a population-based study," in Proceedings of the IEEE Computing in Cardiology Conference (CinC '13), pp. 691-694, Zaragoza, Spain, September 2013.

[16] K. H. W. J. Ten Tusscher, D. Noble, P. J. Noble, and A. V. Panfilov, "A model for human ventricular tissue," American Journal of Physiology-Heart and Circulatory Physiology, vol. 286, no. 4, pp. H1573-H1589, 2004.

[17] K. Tran, N. P. Smith, D. S. Loiselle, and E. J. Crampin, "A thermodynamic model of the cardiac Sarcoplasmic/Endoplasmic $\mathrm{Ca}^{2+}$ (SERCA) pump," Biophysical Journal, vol. 96, no. 5, pp. 20292042, 2009.

[18] M. Makinose, "Possible functional states of the enzyme of the sarcoplasmic calcium pump," FEBS Letters, vol. 37, no. 2, pp. 140-143, 1973.

[19] L. D. Meis and A. L. Vianna, "Energy interconversion by the $\mathrm{Ca}^{2+}$-dependent ATPase of the sarcoplasmic reticulum," Annual Review of Biochemistry, vol. 48, pp. 275-292, 1979.

[20] J. P. Ebus, Z. Papp, R. Zaremba, and G. J. M. Stienen, "Effects of MgATP on ATP utilization and force under normal and simulated ischaemic conditions in rat cardiac trabeculae," Pflügers Archiv, vol. 443, no. 1, pp. 102-111, 2001.

[21] A. F. De Castro, A. Giovanni, J. F. Rodriguez, and J. M. Ferrero, "Dynamic computational simulations of alternans in acute myocardial ischemia," in Proceedings of the 41st IEEE Computing in Cardiology Conference (CinC '14), pp. 877-880, September 2014.

[22] I. Martišiene, J. Jurevičius, R. Vosyliute et al., "Evolution of action potential alternans in rabbit heart during acute regional ischemia," BioMed Research International, vol. 2015, Article ID 951704, 12 pages, 2015.

[23] J. Carro, J. F. Rodriguez, P. Laguna, and E. Pueyo, "A human ventricular cell model for investigation of cardiac arrhythmias under hyperkalaemic conditions," Philosophical Transactions of the Royal Society of London, Series A: Mathematical, Physical and Engineering Sciences, vol. 369, no. 1954, pp. 4205-4232, 2011.

[24] O. Bernus, C. W. Zemlin, R. M. Zaritsky, S. F. Mironov, and A. M. Pertsov, "Alternating conduction in the ischaemic border zone as precursor of reentrant arrhythmias: a simulation study," Europace, vol. 7, no. S2, pp. S93-S104, 2005.

[25] L.-H. Xie, D. Sato, A. Garfinkel, Z. Qu, and J. N. Weiss, "Intracellular Ca alternans: coordinated regulation by sarcoplasmic reticulum release, uptake, and leak," Biophysical Journal, vol. 95, no. 6, pp. 3100-3110, 2008.

[26] Z. Qu, M. B. Liu, and M. Nivala, "A unified theory of calcium alternans in ventricular myocytes," Scientific Reports, vol. 6, Article ID 35625, 2016.

[27] X. Wan, M. Cutler, Z. Song et al., "New experimental evidence for mechanism of arrhythmogenic membrane potential alternans based on balance of electrogenic INCX/ICa currents," Heart Rhythm, vol. 9, no. 10, pp. 1698-1705, 2012. 


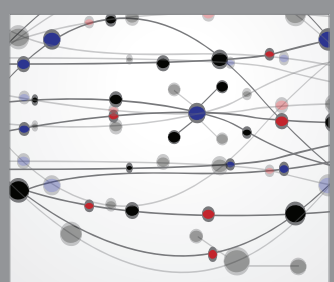

The Scientific World Journal
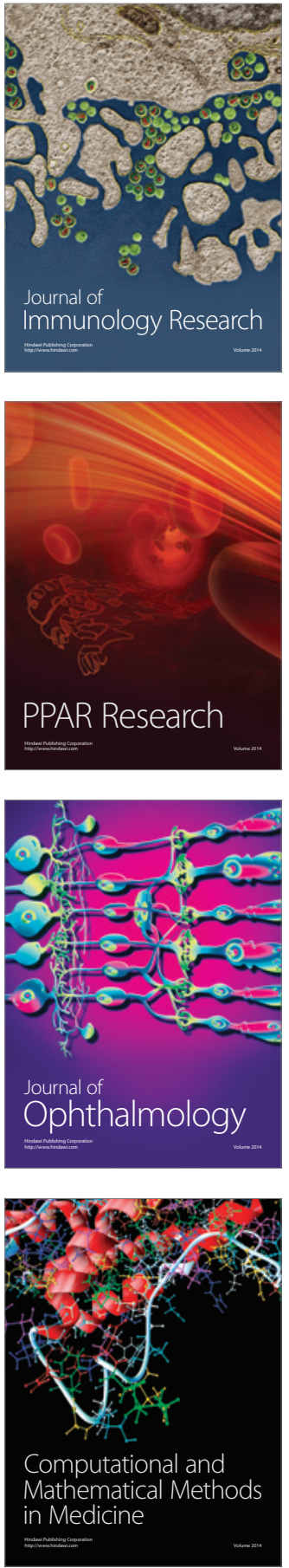

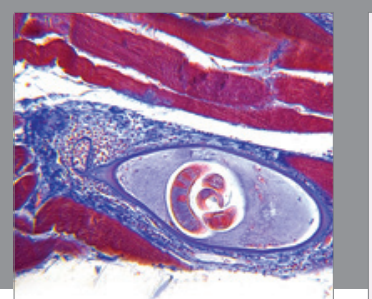

Gastroenterology Research and Practice

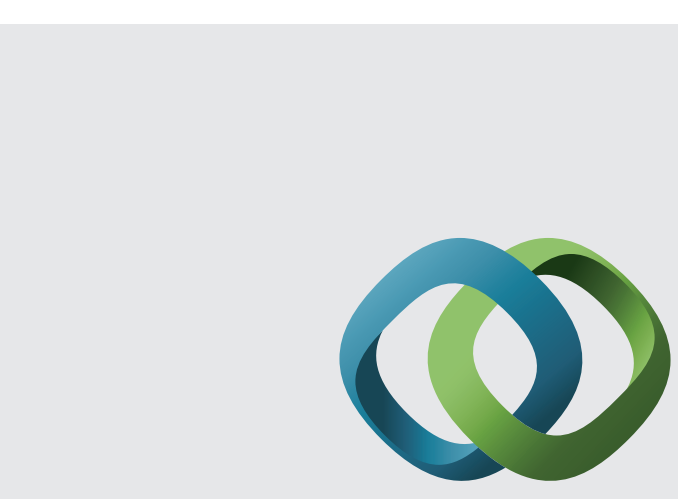

\section{Hindawi}

Submit your manuscripts at

http://www.hindawi.com
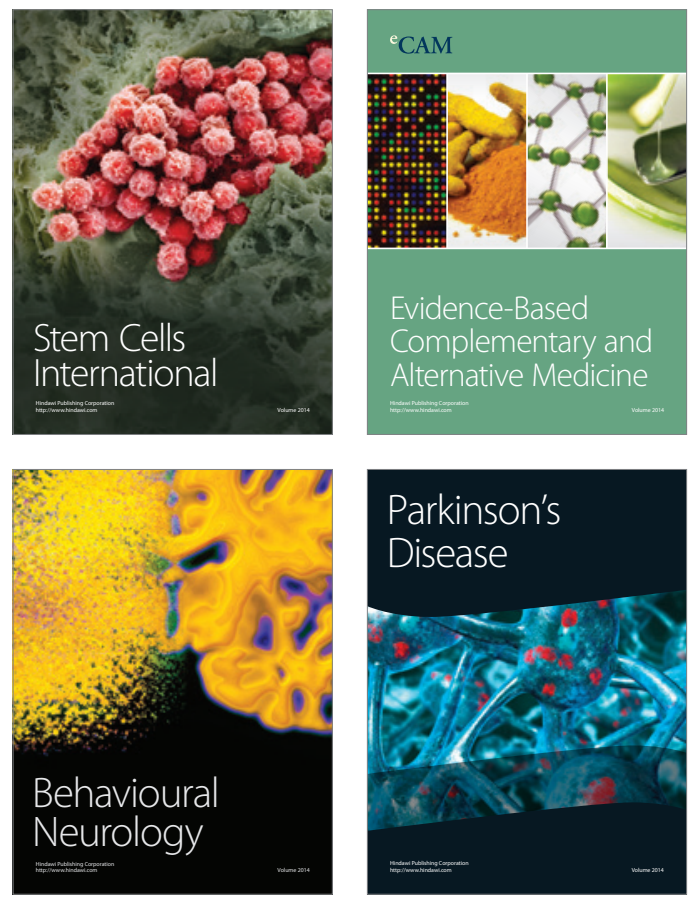
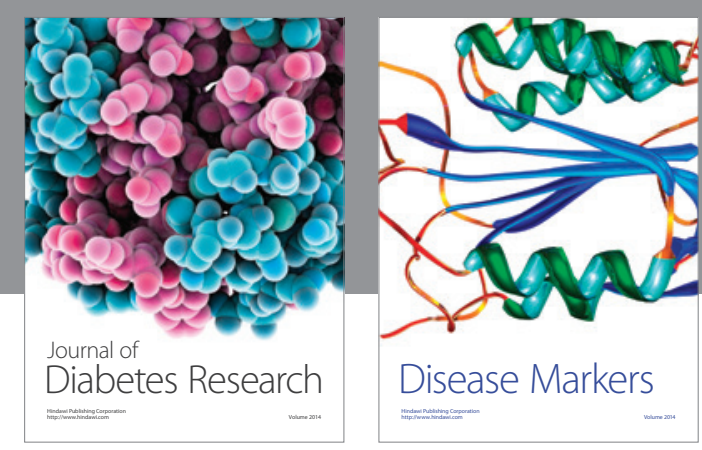

Disease Markers
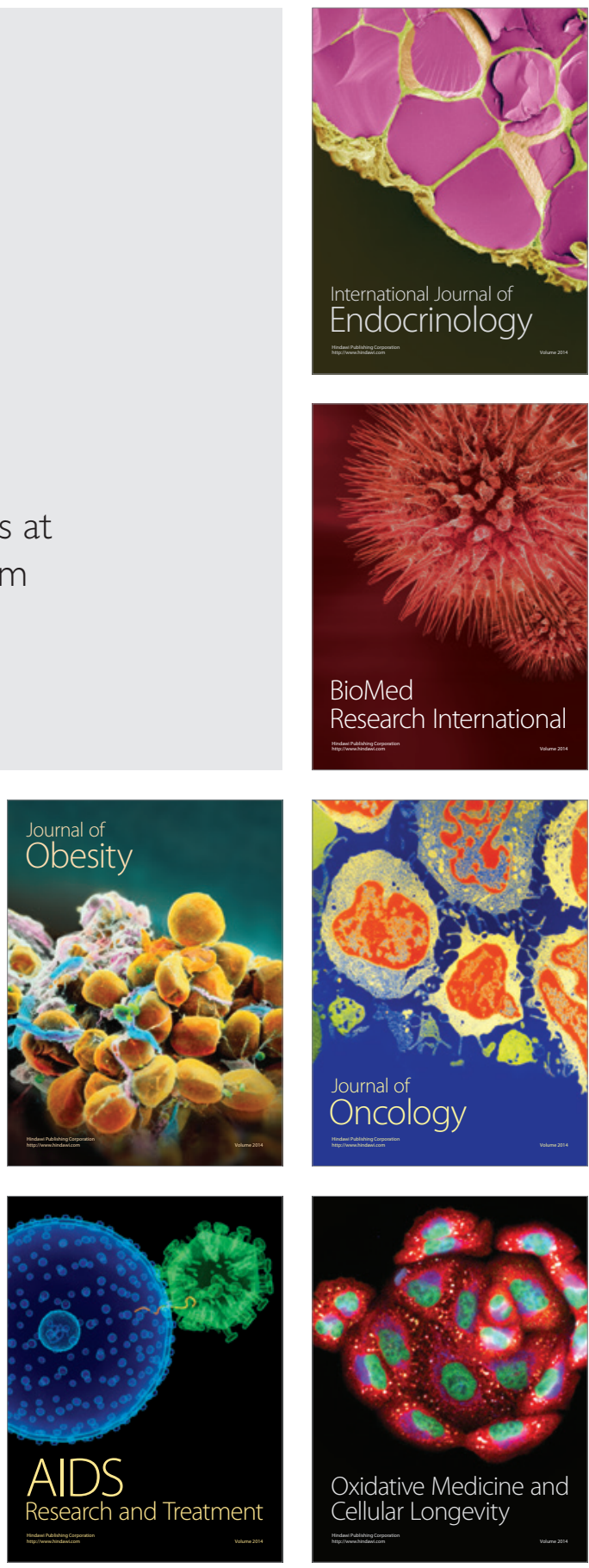